\title{
Sensitization to Airborne Fungal Allergens Associates with Asthma and Allergic Rhinitis Presentation and Severity in the Singaporean/Malaysian Population
}

\author{
Yang Yie Sio • Sze Lei Pang $\cdot$ Yee-How Say $\cdot$ Keng Foo Teh $\cdot$ Yi Ru Wong • \\ Smyrna Moti Rawanan Shah • Kavita Reginald • Fook Tim Chew (D)
}

Received: 22 December 2020/Accepted: 28 January 2021 / Published online: 13 July 2021

(C) The Author(s) 2021

\begin{abstract}
Fungal spores and conidia are the major components of total airspora in the tropical Asia environment, and their sensitization patterns are often associated with allergic diseases such as asthma, allergic rhinitis (AR), and atopic dermatitis. Hence, we recruited a cross-sectional cohort of 9223 Singapore/ Malaysia Chinese adults and assessed their sensitization against Curvularia lunata allergen using the skin prick test approach. A subset of this cohort $(n=254)$ was also screened for specific Immunoglobulin $\mathrm{E}$ (sIgE) titers against a panel of 11 fungal allergens. We found significant association of Curvularia lunata
\end{abstract}

Handling Editor: Sanjay Haresh Chotirmall

Supplementary Information The online version contains supplementary material available at (https://doi.org/10.1007/ s11046-021-00532-6).

Present Address:

Y. Y. Sio · S. L. Pang · Y.-H. Say · F. T. Chew ( $₫)$

Department of Biological Sciences, National University of Singapore, Singapore, Singapore

e-mail: dbscft@nus.edu.sg

Y.-H. Say

Department of Biomedical Science, Faculty of Science, Universiti Tunku Abdul Rahman (UTAR) Kampar

Campus, Kampar, Perak, Malaysia

K. F. Teh · Y. R. Wong · S. M. R. Shah · K. Reginald Department of Biological Sciences, School of Science and Technology, Sunway University, Subang Jaya, Malaysia sensitization with the risk of asthma $(\mathrm{OR}=1.66,95 \%$ CI: $1.17-2.33 ; p=0.00391)$ and $\mathrm{AR}(\mathrm{OR}=1.69$, 95\% CI: $1.18-2.41 ; p=0.00396)$. Among asthmatic patients $(n=1680)$, Curvularia lunata sensitization also increased frequencies of wheezing symptoms $(\mathrm{OR}=1.81,95 \%$ CI: 1.05-2.96; $p=0.0239)$, general practitioner/specialist visits $(\mathrm{OR}=2.37,95 \% \mathrm{CI}$ : $1.13-4.61 ; p=0.0157)$, and other asthma-related exacerbation events $(\mathrm{OR}=2.14,95 \%$ CI: $1.04-4.10$; $p=0.0289$ ). In our serum cohort, sensitization to Aspergillus spp. was the most common fungal sensitization, with $23.6 \%(n=60)$ had a class 3 and above sensitization (positive sensitization; sIgE titers of $>$ $3.5 \mathrm{kU} / \mathrm{L}$ ) against this allergen. Increasing sIgE titer against Aspergillus spp. was also correlated with increased AR risk and AR-related symptoms. In conclusion, our findings emphasize an important role of fungal sensitization in the manifestations of asthma and AR in the Southeast Asian Chinese population.

Keywords Allergy · Fungi - Sensitization · Aspergillus $\cdot$ Curvularia

\section{Introduction}

The worldwide prevalence of allergic diseases, including asthma, allergic rhinitis (AR), and atopic dermatitis (AD), has increased over the past decades 
[1-3]. Sensitizations to dust mites, pollen, and spores have been previously indicated as major triggers of these allergic diseases [4]. AR has been considered as a post-industrial revolution epidemic due to increase in urbanization air pollution [5]. The ongoing fourth industrial revolution will continue to exacerbate allergic diseases due to air pollution and climate change [6]. In the tropical urban environment of Singapore, multiple fungal species including Cladosporium spp., Didymosphaeria spp., Curvularia spp., Drechslera spp., and Pithomyces spp. were the most dominant fungal spore type in the total outdoor airspora and their spores densities were affected by temperature and relative humidity [7].

Here, we report the association of airborne fungal sensitization with allergic disease susceptibility and severity in a Southeast Asian Chinese population. Using a cross-sectional cohort of 9923 Singapore/ Malaysia Chinese adults, we assessed their Curvularia lunata sensitization profile using skin prick test (SPT) and compared across their asthma-, AR-, or ADrelated phenotypes. Using a subset of serum samples, we also evaluated specific Immunoglobulin E (specific $\operatorname{IgE}$ or $\operatorname{sIgE}$ ) titers against multiple fungal allergens presented in the tropical Asia environment and associated them with AR-related phenotypes.

\section{Materials and Methods}

\section{Study Design}

The current study belonged to a part of our ongoing epidemiology study on allergic diseases. Participants were recruited from three locations, including the National University of Singapore (NUS), Universiti Tunku Abdul Rahman (UTAR), and Sunway University (SU), Malaysia. Recruitments were conducted from Aug 2005 to Sep 2019 in NUS, from Feb 2016 to Oct 2018 in UTAR, and on Nov 2019 in SU. Participants were requested to complete an investigator-administered questionnaire collecting information on demographics and medical history, which was based on the Allergic Rhinitis Impact on Asthma (ARIA) [1] and International Study of Asthma and Allergies in Childhood (ISAAC) [8] guidelines.

Participants also underwent a skin prick test (SPT). Our full SPT panel included 2 house dust mite species (Dermatophagoides pteronyssinus and Blomia tropicalis), Curvularia lunata, and Oil palm pollen (Elaeis guineensis). These allergens were previously shown to be the most common inhalant allergen presented in the Singapore environment with high allergenicity [7, 9]. Sensitization to house dust mite has already been discussed in our previous publication [10]. We also observed a separate and independent association between sensitization to oil palm pollen and allergic diseases presentation. This will be discussed in a separate publication. In this manuscript, we focused on Curvularia spp. sensitization because this was one of the most abundant outdoor fungal species as mentioned in our previous publication [7].

Ten milliliters of whole blood samples were collected from a subset of participants in our crosssectional cohort. Serum was then separated via centrifugation at $2600 \mathrm{~g}$ and $4{ }^{\circ} \mathrm{C}$ for $15 \mathrm{~min}$ and measured for sIgE titer against 11 fungal allergens (Aspergillus spp., Candida albicans, Penicillium spp., Trichophyton spp., Saccharomyces cerevisiae, Fusarium spp., Trichoderma viride, Stemphylium botryosum, Cladosporium spp., Malazessia furfur, and Curvularia spp.) using the immuno-dot blot assay, as described previously [11-14]. Sensitization of class 3 and above (sIgE titers of $>3.5 \mathrm{kU} / \mathrm{L}$ ) was considered as positive sensitization. All immune-dot blot measurements were conducted at the same time to avoid batch variation.

Asthma was defined as ever having asthma positively diagnosed by a physician. AR was defined as having at least two major AR-related symptoms that include nasal congestion, rhinorrhea, nasal itching, and sneezing (based on 2008 guidelines set by the ARIA consortium) [1]. Based on the severity of these four AR-related symptoms and an approach that was adopted from the Symptomatic Global Score for seasonal allergic rhinitis [15], a numeric score was also calculated for the AR cases and was categorized into three groups-low: $0-2$, mild: $3-4$, moderatesevere: $>4$. AD was defined as having a persistent itchy rash that affected flexural areas. These allergic conditions (asthma, AR, and AD) were further confirmed by participants having an atopy condition, defined as having a positive SPT reaction toward one of the two common house dust mites, Dermatophagoides pteronyssinus or Blomia tropicalis. Ethnicity was self-identified through a questionnaire and further confirmed through a previously performed principal component analysis [16]. 
Association analysis between fungal sensitization and allergic disease-related phenotypes was performed using independent sample $t$-test or logistic regression analysis in the $\mathrm{R}$ program version 3.6.1 ( $\mathrm{R}$ Foundation for Statistical Computing, Vienna, Austria), and $p<0.05$ was considered as statistically significant.

\section{Results}

Sensitization to the Curvularia is Associated with the Susceptibility and Severity of Asthma and AR

Our cross-sectional cohort comprised 9923 Singapore/ Malaysia Chinese adults (age: $21.90 \pm 4.93$ ), with asthma, AR, and $\mathrm{AD}$ prevalence of $16.9 \%, 32.3 \%$, and $14.4 \%$, respectively (Supplementary Table 1). In this cohort, the sensitization rate to Curvularia lunata was 1.9\% (Supplementary Table 1). A positive SPT reaction to the Curvularia lunata allergen was significantly associated with an increased susceptibility to asthma and AR, but not AD (Table 1). Among asthmatic cases, Curvularia lunata sensitization was also significantly associated with the presence of some recent (past 12 months) asthma symptoms or exacerbation events, which included wheezing, general practitioner/specialist visits, and any asthma-related exacerbation event (Table 1). Besides, when AR patients were classified into three groups based on their symptom scores, a moderate-severe phenotype was also associated with Curvularia lunata sensitization, albeit marginally significant ( $p=0.0555$; Table 1). Additionally, individuals with Curvularia lunata sensitization also had increased risk of having both asthma and AR conditions, as well as both AR and AD conditions (Table 1).

Serum sIgE Titers Against Aspergillus spp. Correspond with the Outcomes and Severity of AR

Next, we collected 254 individuals' serum samples from a subset of our cross-sectional cohort (Supplementary Table 1) and measured sIgE titers against 11 common fungal allergens. Positive sensitization to Aspergillus spp. was observed to be the most common (23.62\%, $n=60$ ) among all the tested fungal allergens (Supplementary Fig. 1). Furthermore, significant pairwise correlations were observed across all the sIgE titers against 11 types of fungal allergens tested. (Pearson's R ranged from 0.283 to 0.721 , all $p<0.0001)$. These include the correlation between sIgE titers against Aspergillus spp. and Curvularia spp. (Pearson's $\mathrm{R}=0.373, p<0.0001$, Supplementary Fig. 2). In this serum cohort, we found that individuals with AR generally had significantly higher Aspergillus spp.-specific IgE levels compared to controls ( $p=0.043$, Fig. 1a), and those with mild AR symptom scores also had significantly higher sIgE levels than those with low AR symptom scores $(p=0.040$, Fig. 1b).

\section{Discussion}

In our Southeast Asian Chinese population, we detected significant associations of fungal sensitization with the risk and severity of asthma and AR. Sensitization to Curvularia lunata allergen increases the risk of asthma and AR, while also increasing the frequencies of wheezing symptoms and exacerbation events among asthmatic patients. Furthermore, sensitization to Aspergillus spp. was the commonest among all tested fungal allergens in our serum cohort, with 23.6\% having positive sensitization. Increasing sIgE titer against Aspergillus spp. was also correlated with increased AR risk and AR-related symptoms.

Our study focused on Chinese individuals who resided in the tropical environment of Singapore and Malaysia. A previous aerobiological survey has revealed a rich fungal airspora present in the Singapore environment [7]. Curvularia lunata is especially prevalent in tropical climatic conditions like Singapore and Malaysia, albeit its spore counts decreased with increases in relative humidity and precipitation [7], while the highest concentrations of Aspergillus fumigatus were observed during monsoon season in a southern tropical Indian region [17]. Individuals residing in this region may therefore likely be sensitized to these fungal allergens and subsequently have increased susceptibility to allergic diseases. In agreement with this, a study has shown an association between Curvularia spp. sensitization and atopic diseases in the Singapore population [9]. By using a large cohort of Singapore/Malaysia Chinese individuals that is independent of the previous cohort [9], we were able to confirm this association and showed that 
Table 1 Associations of Curvularia lunata sensitization with allergic diseases risk and severity in the Singapore/Malaysia Chinese population

\begin{tabular}{|c|c|c|c|c|}
\hline & $\begin{array}{l}\text { Curvularia lunata } \\
\text { Positive SPT } \\
\text { Case/control } \\
\text { (\%Case) }\end{array}$ & $\begin{array}{l}\text { Curvularia lunata } \\
\text { Negative SPT } \\
\text { Case/control } \\
\text { (\%Case) }\end{array}$ & $p$ value & OR $(95 \%$ CI $)$ \\
\hline Asthma (AS) & $50 / 118(29.8)$ & $1630 / 6860(19.2)$ & $0.00391 *$ & $1.66(1.17-2.33)$ \\
\hline Wheezing (past 12 months) & $13 / 32(28.9)$ & $303 / 1269(19.3)$ & $0.0239 *$ & $1.81(1.05-2.96)$ \\
\hline Daytime Asthma attack (past 12 months) & $2 / 40(4.8)$ & $38 / 1194(3.1)$ & 0.474 & $1.70(0.27-5.81)$ \\
\hline Nighttime Asthma attack (past 12 months) & $2 / 40(4.8)$ & $41 / 1186(3.3)$ & 0.170 & $2.35(0.55-6.86)$ \\
\hline School absence due to asthma (past 12 months) & $5 / 37(11.9)$ & $77 / 1148(6.3)$ & 0.240 & $1.87(0.59-4.89)$ \\
\hline GP/specialist Visits due to Asthma (past 12 months) & $11 / 30(26.8)$ & $197 / 1027(16.1)$ & $0.0157 *$ & $2.37(1.13-4.61)$ \\
\hline A and E Admission due to Asthma (past 12 months) & $3 / 39(7.1)$ & $51 / 1161(4.2)$ & 0.537 & $1.50(0.34-4.68)$ \\
\hline Hospitalization due to asthma (past 12 months) & $1 / 38(2.6)$ & 19/1145 (1.6) & 0.912 & $1.12(0.06-5.57)$ \\
\hline Any Exacerbation event $\dagger$ (past 12 months) & $12 / 30(28.6)$ & 213/1012 (17.4) & $0.0289 *$ & $2.14(1.04-4.10)$ \\
\hline Allergic Rhinitis (AR) & $75 / 54(58.1)$ & $3126 / 4043(43.6)$ & $0.00396 *$ & $1.69(1.18-2.41)$ \\
\hline AR symptom scores: low (0-2) & $4(3.1)$ & $155(2.2)$ & ref & \\
\hline AR symptom scores: mild (3-4) & $11(8.5)$ & $452(6.3)$ & 0.739 & $1.09(0.65-1.75)$ \\
\hline AR symptom scores: moderate-severe $(>4)$ & $60(46.5)$ & $2518(35.1)$ & 0.0555 & $1.36(0.99-1.87)$ \\
\hline Atopic Dermatitis (AD) & $36 / 125(22.4)$ & $1392 / 6792(17)$ & 0.102 & $1.37(0.93-1.98)$ \\
\hline $\mathrm{AS}+\mathrm{AR}$ & $24 / 42(36.4)$ & $861 / 3241(21)$ & $0.0491 *$ & $1.70(0.99-2.85)$ \\
\hline $\mathrm{AS}+\mathrm{AD}$ & 11/79 (12.2) & 409/4981 (7.6) & 0.224 & $1.52(0.73-2.86)$ \\
\hline $\mathrm{AR}+\mathrm{AD}$ & $22 / 43(33.8)$ & $804 / 3187(20.1)$ & $0.0174 *$ & $1.90(1.10-3.19)$ \\
\hline $\mathrm{AS}+\mathrm{AR}+\mathrm{AD}$ & 9/34 (20.9) & $261 / 2597(9.1)$ & 0.107 & $1.95(0.81-4.18)$ \\
\hline
\end{tabular}

A and E: accident and emergency department of a hospital; CI: confidence interval; GP: general practitioner; SPT: skin prick test. All data were evaluated based on skin prick test results of the Curvularia lunata allergen in a cross-sectional cohort of Singapore/ Malaysia Chinese individuals $(n=9223)$. $p$ value, odds ratio, and 95\% CI were calculated using a logistic regression analysis with adjustment for age and gender

${ }^{\dagger}$ Any exacerbation event includes school absence, GP/specialist visits, A and E admission, or hospitalization due to asthma exacerbation

${ }^{*} p<0.05$ is considered as significant

Curvularia lunata sensitization is associated with the risk of asthma and AR, as well as asthma-related symptoms and exacerbation events. Besides, our study has further demonstrated significant associations of serum Aspergillus spp.-specific IgE titer with AR risk and severity.

Due to a small sample size of asthma cases in the serum cohort, thus limiting statistical power, we were unable to determine a significant association of $\operatorname{sgE}$ specific to fungal allergens with asthma risk and severity. Nevertheless, Aspergillus spp. sensitization was previously associated with frequent asthma exacerbations and increased corticosteroid requirement in the Singapore population with severe asthma [18]. Increasing serum collection from more asthma cases in future may help to confirm this association. Besides, we have not characterized the role of age in fungal sensitization in our study population. This is because our cohort comprises mostly young participants $(21.90 \pm 4.93$ years old for the cross-sectional cohort, supplementary Table 1) that were recruited from three different universities in Singapore and Malaysia. Although the role of age is unclear, a previous study has observed an increased fungal sensitization rate in subjects of higher age [9]. Further study is therefore required to validate this observation.

In conclusion, the current study has characterized fungal sensitization patterns and showed their association with the development of allergic diseases in the Southeast Asian Chinese population. Further characterizations of these fungal allergenic components may provide a better understanding of the manifestations of 


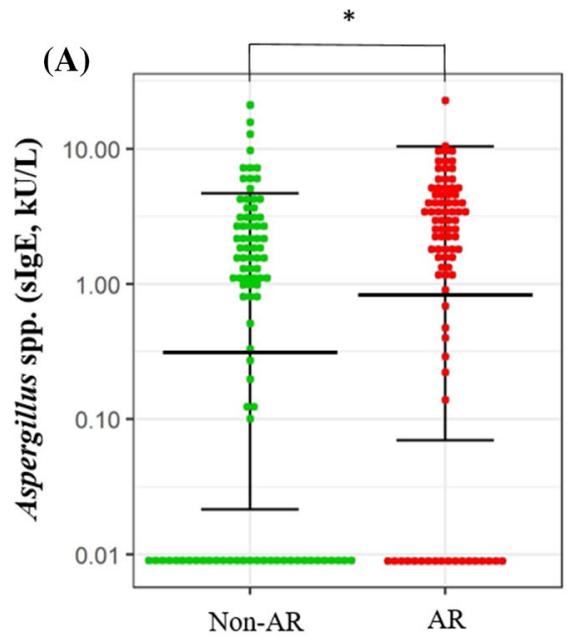

Fig. 1 Association of Aspergillus spp. sensitization with AR risk and severity in the Singapore/Malaysia Chinese population. Serum sIgE titers specific to Aspergillus spp. were compared across a AR and b AR severity based on test subject's AR

allergic diseases in this region, which allow improvement of the current diagnostic and therapeutic approaches of allergic diseases.

Acknowledgements The authors would like to thank the volunteers for participating in this study, Dr. Ramani Anantharaman, Parate Pallavi Nilkanth, Dr. Bani Kaur Suri, and Dr. Sri Anusha Matta for their help with sample collection.

Author Contributions FTC planned and supervised the study. YYS, SLP, YHS, KFT, YRW, SMRS, and KR recruited participants for the study. YYS and SLP planned and conducted the experiments, as well as analyzed the data. YYS wrote the manuscript. All authors reviewed and approved the manuscript.

Funding F.T.C. has received research support from the Singapore Ministry of Education Academic Research Fund, Singapore Immunology Network (SIgN), National Medical Research Council (NMRC) (Singapore), Biomedical Research Council (BMRC) (Singapore), and the Agency for Science Technology and Research (A*STAR) (Singapore); Grant Numbers: N-154-000-038-001, R-154-000-191-112, R-154000-404-112， R-154-000-553-112， R-154-000-565-112， R154-000-630-112, R-154-000-A08-592, R-154-000-A27-597, R-154-000-A91-592, R-154-000-A95-592, BMRC/01/1/21/18/ 077, BMRC/04/1/21/19/315, SIgN-06-006, SIgN-08-020, $\mathrm{NMRC} / 1150 / 2008$, and H17/01/a0/008. The funding agencies had no role in the study design, data collection, and analysis, decision to publish, or preparation of the manuscript.

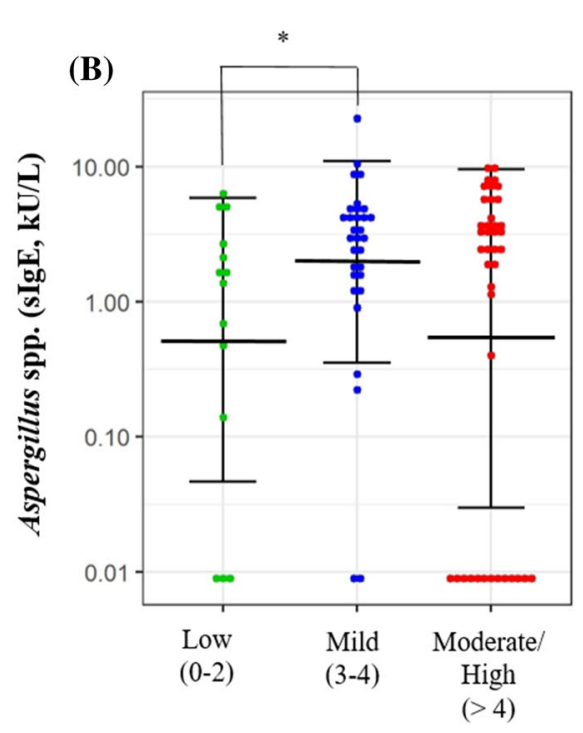

AR Symptom Score

symptom score (low: 0-2, mild: 3-4, and moderate-severe: $>4$ ), using a cross-sectional cohort of Singapore/Malaysia Chinese individuals $(n=254)$. $* t$-test $p$ value $<0.05$

Data Availability All data generated, analyzed, and included in this study are available from the corresponding author (Chew Fook Tim).

\section{Compliance with Ethical Standards}

Conflict of interest The authors declare no competing interests.

Ethical Approval Ethical approval for participant recruitment in Singapore was granted from the Institutional Review Board of NUS (NUS-IRB Ref-Code: 07-023, 09-256, 10-445, 13-075, B-10-343, and H-18-036) and the National Healthcare Group Domain Specific Review Board (B/04/055). While in Malaysia, ethical approval was granted from the Scientific and Ethical Review Committee (SERC) of UTAR (Ref-code: U/SERC/03/2016), as well as from the Research Ethics Committee at Sunway University (SU), Malaysia (Ref. code: SUREC 2019/029). All participants (or parent/guardian for those below 21 years) signed informed consent forms, and the study was conducted in accordance with the Declaration of Helsinki.

Open Access This article is licensed under a Creative Commons Attribution 4.0 International License, which permits use, sharing, adaptation, distribution and reproduction in any medium or format, as long as you give appropriate credit to the original author(s) and the source, provide a link to the Creative Commons licence, and indicate if changes were made. The images or other third party material in this article are included in the article's Creative Commons licence, unless indicated otherwise in a credit line to the material. If material is not included in the article's Creative Commons licence and your intended use is not permitted by statutory regulation or exceeds 
the permitted use, you will need to obtain permission directly from the copyright holder. To view a copy of this licence, visit http://creativecommons.org/licenses/by/4.0/.

\section{References}

1. Bousquet $\mathbf{J}$, et al. Allergic rhinitis and its impact on asthma (ARIA) 2008 update (in collaboration with the World Health Organization, GA(2)LEN and AllerGen). Allergy. 2008;63(86):8-160.

2. Bateman ED, et al. Global strategy for asthma management and prevention: GINA executive summary. Eur Respir J. 2008;31(1):143-78.

3. Asher MI, et al. Worldwide time trends in the prevalence of symptoms of asthma, allergic rhinoconjunctivitis, and eczema in childhood: ISAAC phases one and three repeat multicountry cross-sectional surveys. Lancet. 2006;368(9537):733-43.

4. Kay AB. Allergy and allergic diseases. First of two parts. N Engl J Med. 2001;344(1):30-7.

5. Emanuel MB. Hay fever, a post industrial revolution epidemic: a history of its growth during the 19th century. Clin Allergy. 1988;18(3):295-304.

6. Oláh J, et al. Impact of industry 4.0 on environmental sustainability. Sustainability. 2020;12:4674.

7. Lim SH, et al. Outdoor airborne fungal spores in Singapore. Grana. 1998;37(4):246-52.

8. Asher MI, et al. International study of asthma and allergies in childhood (ISAAC): rationale and methods. Eur Respir J. 1995;8(3):483-91.

9. Chew FT, et al. Evaluation of the allergenicity of tropical pollen and airborne spores in Singapore. Allergy. 2000;55(4):340-7.
10. Andiappan AK, et al. Allergic airway diseases in a tropical urban environment are driven by dominant mono-specific sensitization against house dust mites. Allergy. 2014;69(4):501-9.

11. Gao YF, et al. Identification and characterization of a novel allergen from Blomia tropicalis: Blo t 21. J Allergy Clin Immunol. 2007;120(1):105-12.

12. Kidon MI, et al. Mite component-specific IgE repertoire and phenotypes of allergic disease in childhood: the tropical perspective. Pediatr Allergy Immunol. 2011;22(2):202-10.

13. Chan SL, et al. Nuclear magnetic resonance structure and IgE epitopes of Blo t 5, a major dust mite allergen. J Immunol. 2008;181(4):2586-96.

14. Batard T, et al. Production and proteomic characterization of pharmaceutical-grade Dermatophagoides pteronyssinus and Dermatophagoides farinae extracts for allergy vaccines. Int Arch Allergy Immunol. 2006;140(4):295-305.

15. Rouve $\mathrm{S}$, et al. Numeric score and visual analog scale in assessing seasonal allergic rhinitis severity. Rhinology. 2010;48(3):285-91.

16. Andiappan AK, et al. Evaluating the transferability of Hapmap SNPs to a Singapore Chinese population. BMC Genet. 2010;11:36.

17. Priyamvada $\mathrm{H}$, et al. Seasonal variation of the dominant allergenic fungal aerosols-One year study from southern Indian region. Sci Rep. 2017;7(1):11171.

18. Goh KJ, et al. Sensitization to Aspergillus species is associated with frequent exacerbations in severe asthma. J Asthma Allergy. 2017;10:131-40.

Publisher's Note Springer Nature remains neutral with regard to jurisdictional claims in published maps and institutional affiliations. 